\title{
Contribution à l'étude de l'épidémiologie des bilharzioses humaine et animale en Haute-Casamance (Sénégal) et en Mauritanie
}

\author{
par S. GRETILLAT
}

La première enquête épidémiologique sur la bilharziose vésicale en Haute-Casamance semble être celle de BOUET et ROUBAUD en 1912. Ces auteurs considèrent Kolda comme un important foyer de schistosomiase. BOIRON et KOERBER en 1947, puis DESCHIENS (1951, a et 1951, b), et enfin GAUD en 1955, confirment la forte endémicité bilharzienne de cette région du Sénégal.

Au sujei de la même affection en Mauritanie et dans le bas fleuve Sénégal, LEFROU en 1933, récolte de très nombreux bulins qu'il considère comme étant des Bulınus dybowskyi, dans des trous d'eau des jardins de St-Louis, et pense que certains cas de bilharziose vésicale observés dans cette ville ont été contractés au niveau de ces gîtes.

Pour DESCHIENS (1951), Atar, Tidjidja sur le plateau du Tagant, ef le massif de l'Assaba, ont des mares permanentes (gueltas), qui, avec les nombreux Bulinus qu'elles hébergent, doivent être considérées comme autant de foyers de bilharziose vésicale. En 1955, GAUD met l'accent sur l'importance de cette affection parasitaire dans les mêmes régions, alors que BUTT$N E R$, en 1958, estime que les coutumes et l'économie traditionnelle sont des facteurs très importants dans la dispersion de cette helminthiase en République Islamique de Mauritanie.

L'enquête la plus récente est celle de MARIL.L en 1960. Cet auteur trouve Bulinus s. s. dans de nombreux points d'eau (de la région de Rosso à Atar, ef dans le Tagant, à Tidjidja) mais ne donne

Rev. Elev. Méd. vét. Pays trop., 1963, 16, no 3.

Reçu pour publication : juillet 1963. que très peu de détails sur le taux d'infestation des mollusques, sur leur écologie et ne précise pas quelle est l'espèce vectrice. Il est vrai que le travail de MARILL est axé principalement sur lo recherche de la fréquence et de l'importance de la maladie chez l'homme, plutôt que sur celle des hôtes intermédiaires et sur les conditions de contamination de l'hôte définitif.

De 1960 à 1962, le service d'helminthologie du Laboratoire national de recherches vétérinaires de Dakar a pu effectuer plusieurs enquêtes malacologiques dans les points d'eau de Haute-Casamance, du plateau du Tagant (Moudjeria) et de la région de Rosso, au sujet de l'épidémiologie de la bilharziose des ruminants domestiques et de la distomatose bovine. Parallèlement à ce travail et au cours des très nombreuses dissections de bulins effectuées sur le terrain, il a été possible de recueillir un certain nombre d'observations concernant l'épidémiologie de la bilharziose vésicale humaine.

\section{ENQUETTES MALACOLOGIQUES \\ FAITES EN HAUTE-CASAMANCE (KOLDA) EN JUIN-JUILLET 1960 ET EN MAI 1961}

1. Réseau hydrographique et gîtes à mollusques.

A Kolda, le fleuve Casamance, qui est à environ $50 \mathrm{~km}$ de sa source, n'est en période de basses eaux, de février à juillet, qu'un cours d'eau de queiques dizaines de mètres de large sur 1 à 2,5 mètres de profondeur, le lit étant coupé par endroits par des sevils dont la profondeur de l'eau n'atteint pas $20 \mathrm{~cm}$. 
Une flore aquatique plus ou moins dense, composée surtout de Nymphaea et de Pistio stratiotes, un débit peu important, sur un sol très vaseux, contribuent d̀ l'établissement et à la perennité de nombreux gîtes à mollusques tout au long du fleuve, jusqu'aux environs de DianaMalari à $40 \mathrm{~km}$ en aval de Kolda.

Plus en aval, les eaux ne sont plus douces en raison de l'influence de la marée qui remonte très loin à l'intérieur des terres dans cette région de faible altitude.

En Haute-Casamance, les seuls marigots perennes sont ceux de la rive gauche du fleuve, qui se collectent dans un cours d'eau (marigot de Saré Sara). Ce dernier, se jette dans la Casamance, $20 \mathrm{~km}$ en amont de Kolda et prend sa source sur la frontière sénégalo-guinée portugaise.

Tout cet ensemble hydrographique, marécageux par endroits, est caractérisé par une flore aquatique identique à celle de la Casamance avec de très nombreux gîtes à mollusques.

Durant toute la saison sèche, ces gastéropodes d'eau douce « estivent» dans ces gîtes dans lesquels une eau peu profonde à courant très faible sur fond vaseux et une teneur moyenne de l'eau en matières organiques, leur permettent, sinon de se reproduire, du moins de survivre d'une saison des pluies à l'autre.

Au cours de l'hivernage, de juillet à octobre, les fortes précipitations atmosphériques font déborder la Casamance et ses marigots affluents, mais les gîtes à mollusques, quoique fortement perturbés par le courant, très fort en période de crue, sont, en partie, maintenus par les tiges et les feuilles de nénuphars et de lotus.

C'est ainsi qu'à Kolda, à la suite d'une crue de 2,5 m de la Casamance en juillet 1960, il nous a été possible de faire les observations suivantes :

10 Destruction de certains gîtes repérés la veille de la montée des eaux, avec mollusques emportés à la dérive fixés à des feuilles ou à des racines de Pistia stratiotes, ou a des fragments de bois pourri.

$2^{\circ}$ Densité malacologique au mètre carré considérablement diminuée le lendemain de la pluie.

3048 heures après la crue, les eaux étant descendues de 1 mètre environ, augmentation sensible de la densité malacologique avec nombreux spécimens adultes fixés soit sur des graminées du bord de la rive, solt surtout sur des feuilles de nénuphars du milieu du fleuve.

A ce sujet, nous pensons qu'il est intéressant de signaler que vraisemblablement par un mécanisme d'auto-défense, les feuilles de Nymphoea, dont la face inférieure constitue un support idéal pour les mollusques après avoir été submergées par la crue, émergent au bout de 48 heures, leurs tiges les reliant aux racines fixées au fond du fleuve, s'allongeant démesurément de 3, 4, 5 et même 6 mètres (Observation faite au niveau du pont de Kolda en juillet 1960).

En résumé, en région de Kolda, la Casamance et ses affluents (ceux de sa rive gauche), présentent en de nombreux endroits, des conditions biophysico-chimiques permettant aux gastéropodes d'eau douce de se multiplier pendant la saison chaude et de survivre pendant la saison sèche.

\section{Mollusques d'eau douce récoltés en région de Kolda, dans la Casamance et ses marigots affluents.}

Lanistes adansoni (KOBELT), gros mollusque operculé, est très abondant en saison des pluies le long des rives d̀ fond vaseux (DAGET le signale en 1961 dans tout le bassin de la Gambie).

Cleopatra bulimoides (OLIVIER) est beaucoup plus rare que le précédent. Très abondant dans la Gambie (DAGET, 1961). Dans la Casamance on le trouve fixé aux feuilles et aux tiges de Nymphoed.

Biomphalaria pfeifferi gaudi (RANSON), assez rare, surtout sur le bord des rives où il est fixé sur des fevilles de graminées immergées.

Anisus (Gyroulus) notolensis est très commun dans les fonds de marigots fangeux.

Bulmus senegalensis (MÜLLER), d'après le petit nombre d'exemplaires récoltés, ce mollusque, qui est très fréquent dans les mares du Saloum et du Sénégal oriental, ne semble pas trouver dans les eaux de cette région les conditions d'un biotope idéal.

Lymnoea natalensis caillaudi (BOURGUIGNAT), exemplaires abondants fixés aux feuilles de nénuphars. Hôte intermédicire de Fosciola gigantica COBBOLD 1885, ce mollusque maintient dans cette région du Sénégal un toux élevé d'endémicité distomienne (GRETILLAT, 1961, b). 


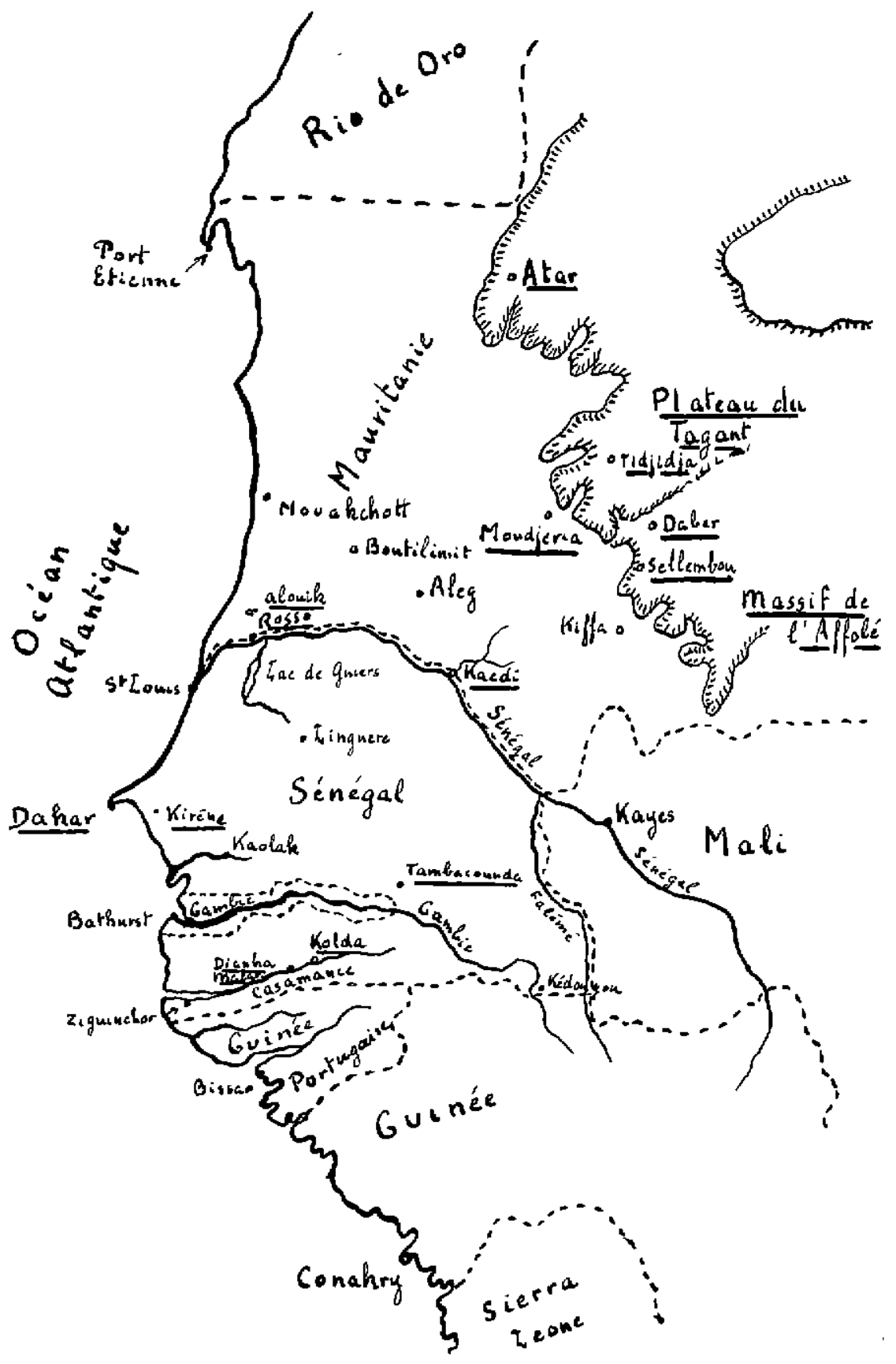

Carte no 1. - Les noms de région ou de localité qui sont soulignés sont ceux mentionnés dans le texte. 


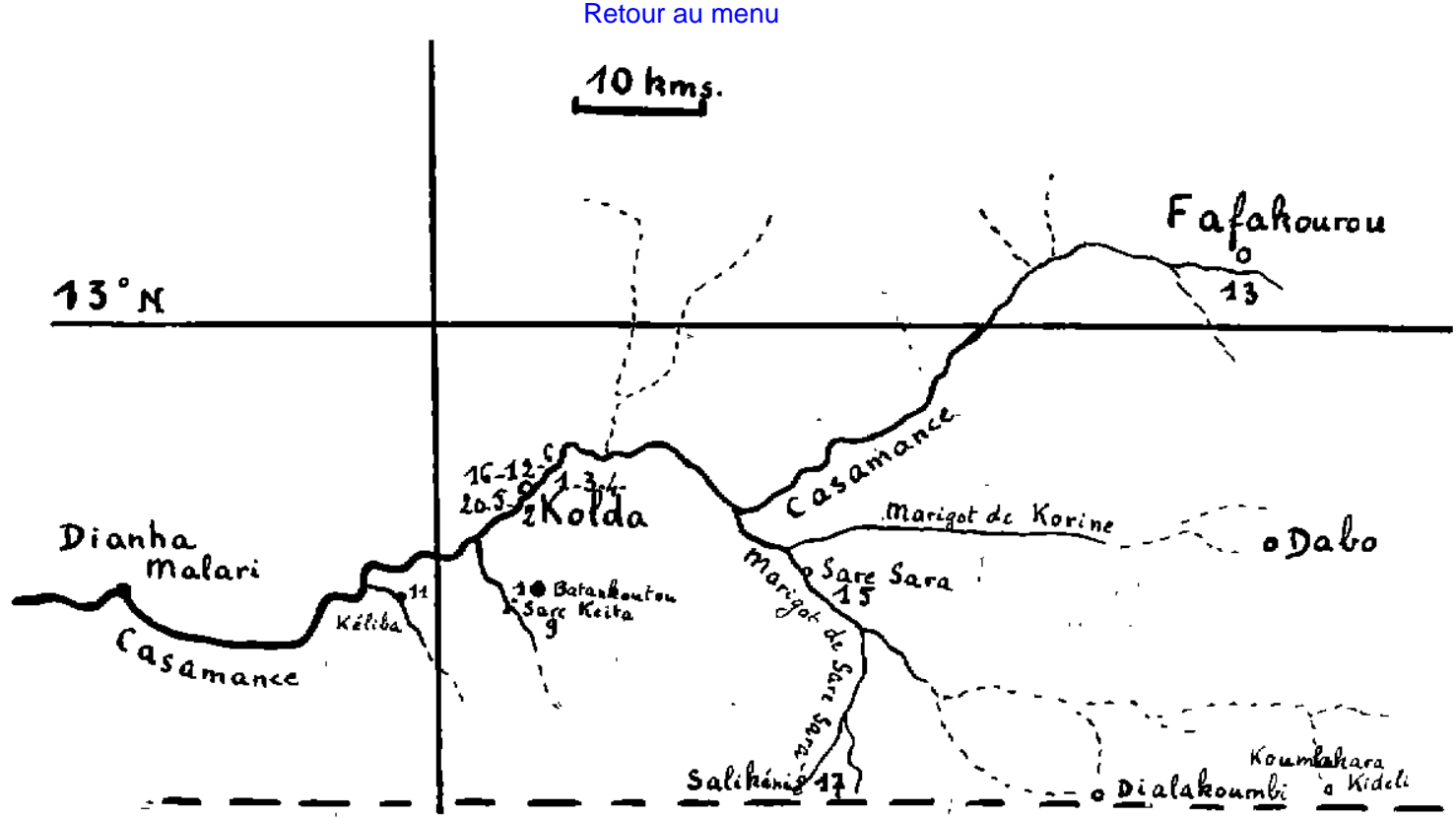

Carte $n^{\circ}$ 2. - Haute Casamance. Région de Kolda. Localités et gîtes à Bulinus jousseoumei. Les chiffres correspondent aux numéros des gîtes indiqués dans le tableau no 1.

TABLEAU PIO I

(Récolte et dissections de Bulinus jousseaumei)

\begin{tabular}{|c|c|c|c|c|c|c|c|c|c|c|c|c|}
\hline $\begin{array}{l}\text { Récaltes } \\
\text { (Dates et } \\
\text { nombre) }\end{array}$ & Gites - Nature & $\begin{array}{l}\text { Densité } \\
\text { malecologi- } \\
\text { que.Par } \mathrm{m}^{2}\end{array}$ & Flore & Tømp. & $\mathrm{pH}$ & Support. & Disge & Ech.' & $\begin{array}{l}\text { farmes } 1 \\
\text { pourcent } \\
\text { Xuph. }\end{array}$ & $\begin{array}{l}\text { arvaire } \\
\text { Pares. } \\
\text { Par. }\end{array}$ & $\begin{array}{l}\text { nature, } \\
\text { Stri. }\end{array}$ & Dìv \\
\hline $\begin{array}{l}1-\text { VII-60 } \\
(70)\end{array}$ & $\begin{array}{l}n^{\circ} \text { 1- Gasamance rive } D \text {.en amont du pont } \\
\text { fond vaseux }\end{array}$ & $5-10$ & ETrentnées & $28^{0}$ & 6,6 & $\begin{array}{c}\text { bois } \\
\text { pourri }\end{array}$ & .10 & 10 & 30 & & 30 & \\
\hline $\begin{array}{l}1-\mathrm{VII}-60 \\
(50)\end{array}$ & $\begin{array}{l}\text { no 2- Casamance sous le pont - } \\
\text { fond plerraux }\end{array}$ & 5 & néent & $27^{\circ}$ & 6,8 & $\begin{array}{l}\text { ciment } \\
\text { du pont }\end{array}$ & 10 & 15 & & & 40 & \\
\hline $\begin{array}{l}1-\text { VIT-60 } \\
(100)\end{array}$ & $\begin{array}{l}n^{0} 3-\text { Cesamance- mllieu du fleuve à } \\
300 \text { m.en amont du pont-vaseux. }\end{array}$ & 5 & $\begin{array}{l}\text { nénupharg } \\
\text { pistia }\end{array}$ & $28^{\circ}$ & 6,7 & $\begin{array}{l}\text { nénuphara } \\
\text { ti.ges et } \\
\text { feuilios. }\end{array}$ & 10 & 5. & & & 40 & 10 \\
\hline $\begin{array}{l}1-\sqrt{I} I-60 \\
(30)\end{array}$ & $\begin{array}{l}\text { no 4- Cesamance-milieu du flieuve en } \\
\text { emont du pant a } 100 \mathrm{~m} \text {-vaseux. }\end{array}$ & 10 & 12. & $30^{\circ}$ & 6,7 & $1 d$. & & 10 & 25 & & 30 & \\
\hline $\begin{array}{l}1-\sqrt{I} I-60 \\
(160)\end{array}$ & $\begin{array}{l}\text { no } 5 \text { - Cesamance au niveau du déversoir } \\
\text { des } \text { î.C. publics - vaseux. }\end{array}$ & 30 & id. & $30^{\circ}$ & 6,6 & Id. & 80 & & & & & \\
\hline $\begin{array}{l}1-V I I-60 \\
(125)\end{array}$ & $\begin{array}{l}n^{0} \text { 6- Casamance rive G. en amont du } \\
\text { pont. Fond plus ou molna vagowx. }\end{array}$ & 20 & ETaminées & $27^{\circ}$ & & $\begin{array}{l}\text { bois } \\
\text { poursi }\end{array}$ & 75 & & & & 5 & \\
\hline $\begin{array}{l}3-\operatorname{VII}-60 \\
(30)\end{array}$ & $\begin{array}{l}\text { no 9- Serekeite } 6 \text { kous de Kolde - } \\
\text { marigot en crue - Fand veseux. }\end{array}$ & 2 & plstia & $28^{\circ}$ & $\overline{6,8}$ & $\begin{array}{l}\text { bols } \\
\text { pourri }\end{array}$ & & & 10 & 10 & 25 & 15 \\
\hline $\begin{array}{l}3-V I \dot{I}-60 \\
(50)\end{array}$ & $\begin{array}{l}\mathrm{n}^{0} 10 \text { - Bantankounto - } 14 \mathrm{kms} \text { de Kolda } \\
\text { tete de marlgot - fond raseix. }\end{array}$ & 5 & $\begin{array}{l}\text { pistia et } \\
\text { nénuphars }\end{array}$ & $27^{\circ}$ & 6,8 & nénupherg & 10 & & & & 25 & \\
\hline $\begin{array}{l}3-V I I-60 \\
(20)\end{array}$ & $\begin{array}{l}\pi^{\circ} 11-\text { Keliba - } 15 \text { kns de Kolda - } \\
\text { marl got en crue - fond plerreux. }\end{array}$ & 1 & $\begin{array}{l}\text { 1d. }+ \\
\text { carex. }\end{array}$ & & ، & Id. & & & 10 & & 25 & \\
\hline $\begin{array}{l}4-\bar{v}=62 \\
(150)\end{array}$ & $\begin{array}{l}n^{\circ} t \overline{2}-\text { Kolda - Casamance en amont du } \\
\text { pont - fond raseux. }\end{array}$ & 10 & nénuphars & $2 \overline{0}$ & 6,1 & némupherg & 30 & & & & 40 & 10 \\
\hline $\begin{array}{l}5-7-62 \\
(24)\end{array}$ & $\begin{array}{l}\text { no 13- Fafekourou ì } 50 \mathrm{kmg} \text { de Kolda - } \\
\text { Sources de la Casamance - Fond vaseux. }\end{array}$ & 1 & $\begin{array}{l}\text { 1d. }+ \\
\text { jancs. }\end{array}$ & $26^{\circ}$ & 5.9 & id. & 10 & & & & 20 & \\
\hline $\begin{array}{l}5-\sqrt{-62} \\
(145)\end{array}$ & $\begin{array}{l}\pi^{\circ} 15-\text { Marigot de Sere-Sere } 20 \text { kns de } \\
\text { Kolda - fond vaseux. }\end{array}$ & 15 & $\begin{array}{l}\text { nénuphara } \\
\text { pistila }\end{array}$ & $27^{\circ}$ & 5.7 & $\begin{array}{l}\text { Ménuphara } \\
\text { feuzlles } \\
\text { pois pourrel }\end{array}$ & 40 & & & 10 & 15 & \\
\hline $\begin{array}{l}7-V-62 \\
(300)\end{array}$ & $\begin{array}{l}\text { no }^{0} \text { 16- Kolda Comemance } 300 \mathrm{~m} \text { en amont } \\
\text { du pont } \sim \text { fond vaseux. }\end{array}$ & 5 i 6 & id. & $28^{\circ}$ & 6,3 & nénuphare & 30 & & & 50 & 10 & \\
\hline $\begin{array}{l}10-\sqrt{1}-62 \\
(130)\end{array}$ & $\begin{array}{l}n^{\circ} \text { 17- Saljkenté - saurces du marigot } \\
\text { de Sare-Sera - terraln marécageux. }\end{array}$ & 10 \& 15 & $\begin{array}{l}\text { nénuphax日 } \\
+ \text { lotu2s }\end{array}$ & $27^{\circ}$ & 5,9 & $\begin{array}{l}\text { aénuphare } \\
\text { et lotus }\end{array}$ & 10 & & & 10 & 10 & \\
\hline $\begin{array}{l}10-y-62 \\
(18)\end{array}$ & $\begin{array}{l}\text { no 18- Marlgot de Koumba Kara-K1déli - } \\
40 \text { kme de Kolda, sur la frontière Séné- } \\
\text { galo-guiné portugalse.fond vaseux. }\end{array}$ & + d 2 & $1 d$. & $28^{\circ}$ & 5,7 & $1 d$. & 5 & & & & 10 & \\
\hline $\begin{array}{l}1 i-\sqrt{-62} \\
(95)\end{array}$ & $\begin{array}{l}\text { no } 20 \text { Kolda Casamence liau de baigne- } \\
\text { de dso enfants. }\end{array}$ & 5 à 10 & $\begin{array}{l}\text { nénuphers } \\
\text { lotus }\end{array}$ & $29^{\circ}$ & 6,2 & id. & 20 & & & 35 & 20 & 5 \\
\hline
\end{tabular}

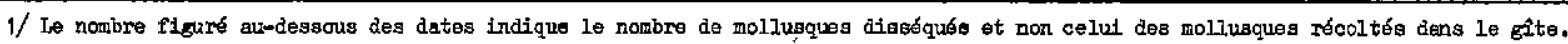

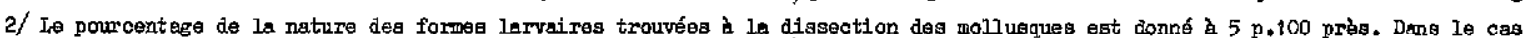
d'association de deur formes larvairee différentes chez un mêne bulin, il n'eat pas tenu compte dens le calcul du pourcentage d'Infestation d'une telle perticularité.

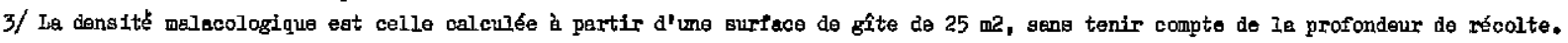

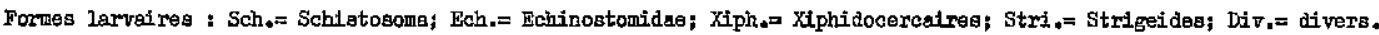


Butinus (Physopsis) jousseaumei (DAUTZEMBERG) tout comme Bulinus guernei DAUTZEMBERG, dont il est très voisin, est au Sénégal l'hôte intermédiaire de la bilharziose vésicale humaine et de la schistosomiase bovine. On le trouve en abondance dans la Casamance et ses affluents, fixé aux feuilles et aux tiges de Nymphaeo ef de lotus ainsi que sur les feuilles d'arbres tombées dans le fleuve et en état de décomposition plus ou moins avancée.

\section{Résultats trouvés à la récolłe et à la dissection des mollusques.}

Bulinus jousseaumer étant le seul gastéropode en cause dans le cas qui nous intéresse, nous ne donnerons sous forme de tableau que les conditions du biơope où a été opérée la récolte, et la nature des formes larvaires trouvées à la dissection du gastéropode vecteur.

\section{Inferprétation des résultats obtenus à la dissection des Bullnus jousseaumei récoltés au cours des enquêtes malacologiques faites en Haute Casamance en juillet 1960 et en mai 1962}

Les formes larvaires de Schistosoma mises à part, le tableau no 1 indique un fort pourcentage d'infestation par furcocercaires de Strigeidaea (Stri.) reconnaissables d̀ la présence d'un pharynx en arrière de la ventouse orale.

Dans les moilusques disséqués provenant des gîtes no 16 et 20,40 p. 100 environ des spécimens infestés par des formes larvaires de Schistosoma l'étaient aussi par des rédies et des cercaires d'échinostomes. Cette association qui paraît assez fréquente chez $B$. jousseaumeı mérite d'être signalée car elle montre qu'un même gastéropode peut simultanément servir d'hôte intermédiaire à deux trématodes appartenant à deux familles totalement différentes, sans qu'apparemment il y ait incompatibilité dans le développement de l'une et de l'autre «parthenita». BRUMPT en 1930 avait déjà signalé cette possibilité d'infestation double chez un même mollusque, mais nous pensons qu'il la considérait comme rare alors qu'elle semble fréquente chez B. jousseaumei en région de Kolda.

Quant aux formes larvaires de Schistosomo trouvées au cours de ces nombreuses dissections (1487, au cours des deux enquêtes) elles méritent comme nous allons le voir, d'attirer quelques remarques.

En effet, si en juilleł 1960, en disséquant les bulins de Haute-Casamance nous avions été frappés par les caractères aberrants des stades larvaires précédant la furcocercaire, par contre en mai 1962, chez 852 exemplaires disséqués, il nous a été impossible de mettre en évidence les sporocystes de Schistosoma haematobium (BILHARZ, 1858) ou ceux de Schistosoma bovis, alors que nous trouvions uniquement chez ces mollusques des formes larvaires identiques à celles que nous avons décrites (GRETILLAT, a et c) pour Schistosoma curassoni Brumpt, 1931.

Afin de prouver qu'en Haute-Casamance le schistosome agent causal de la bilharziose génitourinaire humaine était bien comme en région de Dakar Schistosoma curassoni, nous avons procédé au cours de 1953 à neuf essais d'infestation expérimentales de bulins neufs avec des miracidia éclos d'œufs récoltés dans. des urines d'écoliers bilharziens de Kolda (50 enfants environ).

Au total 787 mollusques appartenant aux espèces suivantes Bulinus guernei, Bulinus truncatus rohifsi et Bulinus contortus (Souche provenant du sud de la Corse), ont servi à cette experience. Les taux d'infestation ont été variables suivant les espèces et les conditions de l'expérimentation (18 à 60 p. 100).

Les résultats suivants ont été obtenus :

10 Toutes les formes larvaires observées à la dissection des bulins ont les caractères morphologiques de celles de Sch. curossoni.

$2^{\circ}$ Bulinus truncatus rohlfsi est meilleur hôte intermédiaire que Bulinus guernei.

30 Bulinus contortus (souche de Corse) peut être infesté expérimentalement par les miracidia de Sch. curassoni et permet l'évolution des formes larvaires de ce schistosome.

40 Les furcocercaires produites obtenues expérimentalement avec ces moilusques d'élevage à partir de souches de schistosame humain de la région de Kolda sont capables d'infester des petits ruminants chez lesquels on retrouve à l'autopsie, 2 mois après l'ingestion des furcocercaires, des vers adultes dans leurs veines mésentériques (Expérience faite sur deux jeunes mou- 
tons neufs provenant de la ferme de Sangalkam où il n'existe oucun gîte à bulins).

Les schistosomes adultes ainsi obtenus ayant les caractères morphologiques de l'espèce Sch. curasson, ces expériences confirment celles réalisées sur deux moutons et une chèvre infestés expérimentalement en mai 1962 avec une souche de schistosome humain de la région de Dakar (GRETILLAT 1962, b).

En résumé, en Haute-Casamance, comme aux environs de Dakar, ef dans le Cayor à Kirène, c'est Sch. curasson! qui est l'agent causal de la bilharziose génito-urinaire humaine ef de la bilharziose des ruminants domestiques, qui ne sont qu'une seule et même zoonose que nous avons appelée en raison de sa répartition géographique «Bilharziose Ouest-Africaine » (GRETILLAT, 1962, b).

\section{Taux d'endémicité bilharzienne et principaux foyers d'infestation dans la région de Kolda.}

Si l'on se réfère aux statistiques du service de Santé de Kolda, ce sont principalement les enfants qui sont atteints de bilharziose vésicale avec hématurie plus ou moins prononcée, 70 à 80 p. 100 des enfants de 3 à 15 ans de cette ville seraient parasités.

Au cours de ces deux enquêtes, nous avons pu constater que le bief de 150 ḋ 200 mètres en amont du pont était un lieu de baignade très fréquenté pendant toute la saison sèche et une partie de l'hivernage par les jeunes de Kolda qui s'y ébattent au cours des heures chaudes de la journée. Or, les dissections de bulins montrent que cette partie de la Casamance est très infectée par Schistosoma curasson dont les furcocercaires doivent sortir en abondance quand l'eau est surchauffée par le soleil.

Au sujet du taux d'infestation des ruminants, les observations faites en 1962 par le service de l'Elevage de Kolda aux abattoirs de cette ville, sont les suivantes:

Bovins :

Forte infestation ............. 10 ¿ 15 p. 100 infestation moyenne......... 20 p. 100 Faible infestation ........... 40 p. 100

Ovins et caprins :

Infestation moyenne......... 30 d̀ 35 p. 100
5. Moyens prophylactiques à mettre en ceuvre pour abaisser le taux d'endémicité bilharzienne en Haute-Casamance.

Le traitement systématique de tous les malades par les produits antibilharziens qu'offre la pharmacopée actuelle est du domaine du clinicien et au point de vue pratique ne peut avoir que peu d'incidence sur l'abaissement du łaux d'endémicité bilharzienne.

En effet, il subsiste pour assurer la perennité de l'espèce parasite, tous les sujets non traités ne présentant pas de bilharziose clinique parce que faiblement parasités mais qui sont des disséminateurs d'œufs.

D'autre part, et c'est le cas pour toutes les zoonoses, le schistosome en cause à Kolda étant un parasite de l'Homme ef des ruminants domestiques, ce sont ces derniers qui représentent le plus gros « réservoir de virus » qu'il est difficile, voire impossible de supprimer, le traitement antibilharzien de tous les ruminants de la région étant une chose impensable tant au point de vue économique que pratique.

II reste donc la destruction de l'hôte intermédicire par des produits molluscicides.

A ce sujet, l'assainissement d'un bief de plusieurs kilomètres en amont et en aval de Kolda par destruction de tous les gîtes à mollusques est une opération d̀ renouveler annuellement puisque un repeuplement malacologique par l'amont aura lieu l'année suivante au cours de la saison des pluies.

Par contre, une campagne antimollusques comportant l'assainissement de la Casamance depuis sa saurce jusqu'à Dianha-Malari, ainsi que celui de ses marigots affluents, amènerait une baisse considérable du taux d'endémicité bilharzienne de toute cette région du Sénégal.

\section{ENQUETES MALACOLOGIQUES FAITES EN MAURITANIE EN DÉCEMBRE |96| ET EN FÉVRIER 1962}

\section{D) RÉGION DE MOUDJERIA (PLATEAU DU TAGANT)}

Les résultats de l'enquête malacologique que nous avons faite dans cette partie de la Mauritanie au sujet de la schistosomiase bovine nous 
permettent de donner quelques précisions en ce qui concerne la faune malacologique des points d'eau et son incidence sur le taux élevé d'endémicité bilharzienne (bilharziose vésicale humaine) existant dans cette région (DESCHIENS, 1951 ; GAUD, 1955; MARILL, 1960).

Le plateau du Tagant est une vaste région d'aspect chaotique, à sol rocheux, d'altitude variable $(200$ à $400 \mathrm{~m})$; se continuant à l'est par le Massif de l'Assaba ef bordé au sud et à l'ouest par une falaise de 200 mètres environ qui tombe à pic sur une région de dunes (A'eg, Boutilimit), de faible altitude située au nord de la boucle du Sénégal.

La seule végétation que l'on trouve sur ce plateau est représentée par quelques épineux, des Euphorbiacées et quelques touffes de graminées poussant entre d'énormes masses rocheuses.

La densité de population est très faible, et les campements de pasteurs nomades possédant ânes, moutons, chèvres et bœufs, en nombre parfois important, sont installés à proximité des mares permanentes de montagne ou «gueltas» autour desquelles poussent des paimiers dattiers et où la culture du mil est possible.

Ces «gueltas» qui sont alimentées par des sources jaillissant de la montagne, sont à des altitudes variables : 200 à 350 mètres. Ce sont de simples poches d'eau à fond rocheux où se rassemblent les eaux qui se perdent par la suite dans le sable. Situées toujours à l'origine d'une ou plusieurs sources, elles ne peuvent être assimilées à des lits d'oueds. Ne tarissant jamais en saison sèche, elles représentent les seuls points d'eau pour la population et les animaux domestiques dans cette partie de la Mauritanie où en raison de la nature du sol, il est pratiquement impossible de creuser des puits.

Très éloignées les unes des autres, d'abord difficile (pour accéder à la plupart de ces mares, il est nécessaire de parcourir parfois plusieurs kilomètres à pied en terrain montagneux), if nous a seulement été possible d'examiner la faune malacologique de deux de ces gueltas de montagne. D'autre part, le désir de ramener du matériel malacologique vivant pour le mettre en élevage au Laboratoire à Dakar, nous a contraint à limiter le nombre de mares prospectées.

Le tableau $n^{0} 2$ montre que dans les gueltas de Daber $(70 \mathrm{~km}$ au Sud-Est-Est de Moudjeria), gîte $n^{\circ} 2$, et de Sellembou (12 km de Moudjeria), gîte no 3, les seuls mollusques récoltés sont des Bulinus truncatus rohlfsi (CLESSIN)*.

Cette espèce a été signalée du Sahara, du Malı, du Cameroun et du Ghana ***.

Dans ces gueltas qui sont des points d'eau complètement isolés au point de vue hydrographique, dans une contrée aride et sèche, ces gastéropodes représentent peut-être une faune relicte.

\section{Résultats trouvés à la dissection des Bulinus trun- catus rohlfsi récoltés dans ces deux gueltas.}

lls sont consignés dans le tableau no 3 (100 exemplaires disséqués pour chaque gîte).

Au sujet de la nature des formes larvaires de Schistosoma trouvées à la dissection, il nous a été impossible de mettre en évidence celles de Schistosoma haematobium ou de Sch. bovis. Par contre un pourcentage appréciable de bulins était parasifé par des éléments identiques aux formes évolutives de Schistosoma curossoni.

En conséquence, comme nous venons de le signaler plus haut pour la bilharziose vésicale humaine en Haute-Casamance, et d'après les résultats que nous avons obtenus au laboratoire avec des souches de schistosomes humains, la bilharziose vésicale existant au Tagant serait la bilharziose Ouest-Africaine d̀ Sch. Curassoni avec comme principal réservoir de parasites les bovins, ovins et caprins.

La grande réceptivité de $B$. truncotus rohlfsi aux miracidia de Sch. curasson! (GRETILLAT, 1962 , a) alliée à la forte densité malacologique des mares et au très fort pourcentage d'infestation des ruminants domestiques de la région, explique le taux élevé d'endémicité bilhorzienne des habitants qui s'infestent en utilisant l'eau de ces gueltas comme boisson. A Kaédi et à Moudjeria sur des bovins, ovins et caprins abattus pour 'a boucherie et provenant du Tagant, nous avons pu constater en 1960 et 1962 des infestations massives par Sch. curassonı chez 70 à 80 p. 100 des animaux avec des lésions hépatiques de nécrose ef de cirrhose.

* Le professeur G. RANSON du Muséum d'histoire naturelle de Paris nous a détermıné ces deux souches à l'cide de 50 exemplaıres d'élevage.

** G. RANSON, communication personnelle. 
TABLEAU n 2. - Gîles à mollusques d'eau douce prospectés dans le Tagant et la région de Rosso (Mauritanie).

\begin{tabular}{|c|c|c|c|c|c|c|c|c|c|c|}
\hline \multirow[b]{3}{*}{ Dates } & \multirow[b]{3}{*}{ Gîtes-Nature } & \multirow[b]{3}{*}{ Flore } & \multirow[b]{3}{*}{ Temp. } & \multirow[b]{3}{*}{$\mathrm{pH}$} & \multicolumn{6}{|c|}{ Mallusques récoltés } \\
\hline & & & & & \multicolumn{2}{|c|}{ B. truncatus } & \multicolumn{2}{|c|}{ B. guernei } & \multicolumn{2}{|c|}{ B. senegalensis } \\
\hline & & & & & $\begin{array}{c}\text { densité } \\
\text { par } \\
\text { mètre } \\
\text { carré }\end{array}$ & support & $\begin{array}{l}\text { densité } \\
\text { par } \\
\text { mètre } \\
\text { carré }\end{array}$ & support & $\begin{array}{c}\text { densilé } \\
\text { par } \\
\text { mètre } \\
\text { carré }\end{array}$ & support \\
\hline $13-12-61$ & $\begin{array}{l}n^{\circ} 1 \text { Moudjeria - sources ali- } \\
\text { mentant la ville - fond ro- } \\
\text { cheux }\end{array}$ & Lemna & $26^{\circ}$ & 5,9 & & & & & 5 & rochers \\
\hline $14-12-61$ & $\begin{array}{c}\text { no }^{\circ} \text { Guelta de Daber }(70 \mathrm{~km} \\
\text { de Moudjeria) fond rocheux }\end{array}$ & néant & 240 & 5,7 & 20 à & rochers & & & & \\
\hline $15-12-61$ & $\begin{array}{l}n^{0} 3 \text { Guelta de Sellembou (12 } \\
\mathrm{km} \text { de Moudjeria) fond ro- } \\
\text { cheux }\end{array}$ & néant & $26^{\circ}$ & 6,0 & 50 & rochers & & & & \\
\hline $12-11-62$ & $\begin{array}{l}n^{\circ} 4 \text { Marigot de Rosso - fond } \\
\text { vaseux }\end{array}$ & $\begin{array}{l}\text { nénu- } \\
\text { phars }\end{array}$ & 240 & 6,0 & & & 20 & $\begin{array}{l}\text { nénu- } \\
\text { phars } \\
\text { vase }\end{array}$ & 5 & nénuphars \\
\hline $13-11-62$ & $\mid \begin{array}{l}n^{0} 5 \text { Lac d'Aoulik (25 km de } \\
\text { Rosso) marécageux }\end{array}$ & $\begin{array}{l}\text { joncs } \\
\text { nénu- } \\
\text { phars }\end{array}$ & 240 & 5,8 & & & 5 & $\begin{array}{l}\text { nénu- } \\
\text { phars }\end{array}$ & 1 c̀ 2 & nénuphors \\
\hline $13-11-62$ & $\begin{array}{l}\text { no } 6 \text { Mare à } 4 \text { km du Lac d'Aou- } \\
\text { lik }\end{array}$ & $\begin{array}{l}\text { nénu- } \\
\text { phars }\end{array}$ & $26^{\circ}$ & 6,0 & & & 5 & $\begin{array}{c}\text { nénu- } \\
\text { phars }+ \\
\text { vase }\end{array}$ & & \\
\hline $13-11-62$ & $\begin{array}{l}\text { no } 7 \text { Marigot de Garak (12 km } \\
\text { de Rosso) fond vaseux }\end{array}$ & $\begin{array}{l}\text { nénu- } \\
\text { phars }\end{array}$ & $26^{\circ}$ & $\mid \begin{array}{l}5,8 \text { d̀ } \\
6,0\end{array}$ & & & 5. & $\begin{array}{l}\text { nénu- } \\
\text { phars }\end{array}$ & & \\
\hline $14-11-62$ & $\begin{array}{l}\text { na } 8 \text { Marigot de Rosso (digue) } \\
\text { fond vaseux }\end{array}$ & id. & $27^{\circ}$ & 6,2 & & & 20 & $\begin{array}{l}\text { id. }+ \\
\text { vase }\end{array}$ & 5 & nénuphars \\
\hline Pour n & $\begin{array}{l}\text { as surcharger le tableau, il n'est } \\
\text { rs des gîtes no } 4,5,6 \text { et } 7 \text { de la }\end{array}$ & tpas fait & it men & ion de & très n & breux & isus $n$ & ensis & oltés st & rles fevilles \\
\hline
\end{tabular}

Moyens prophylactiques à mettre en œuvre pour abaisser le taux d'endémicifé bilharzienne en région du Tagant.

Le faible volume des gueltas (500 à $2.000 \mathrm{~m}^{3}$ d'eau en moyenne), l'absence de courant, plaident en faveur de leur assainissement par épandage d'un produit molluscicide. Cette intervention, peu coûteuse quant au prix de revient des faibles quantités de produit à employer, demande cependant une étude spéciale au sujet des modes de transport à utiliser pour amener sur place les utilisateurs et le produit antimollusques.

Très éloignées les unes des autres, il n'existe parfois qu'un sentier chamelier pour $y$ accéder, becucoup de temps et d'endurance seront exigés des équipes mobiles chargées du traitement de ces gueltas. Par contre, quoique très onéreux, il semble que l'hélicoptère puisse rendre possible une intervention utile tant pour la santé publique que pour l'élevage.

\section{Ii) RÉGION DU bAS FLEUVE SÉNÉGAL (ROSSO)}

Dans cette partie de la Mauritanie, deux sortes de points d'eau sont à considérer : les marigots affluents du Sénégal et communiquant avec lui en hivernage, mais restant en eau pendant toute la saison sèche, les lacs dont l'étendue et l'éloignement du fleuve en font des biotopes un peu particuliers et qui ne sont parfois que de simples collections d'eau plus ou moins sau mâtres dépourvues de faune malacologique. 
a) Les marigots affluents du Sénégal oux alentours de Rosso.

Ces cours d'eau d'importance variable communiquent avec le fleuve en saison des pluies, puis leur niveau baisse considérablement au cours de la saison sèche d'octobre à juillet. lis sont réduits en février-mars à l'état d'étangs ou de mares de plus ou moins grande importance où poussent en abondance des Nymphoea, des lotus, des Pistia ef quelques Cypéracées. La plupart d'entre eux servent à l'abreuvement des animaux domestiques ef très souvent à l'alimentation en eau des habitants de la banlieve immédiate de Rosso. Leur fond est en général très vaseux et comme l'indique le tableau $n^{\circ} 2$ ils constituent d'importants gîtes à mollusques d'eau douce.

b) Les lacs ef marigots des régions nord-auest ef nord-est de Rosso.

Dans la partie de la Mauritanie s'étendant au nord-ovest de Rosso entre la boucle du Sénégal et la mer, existe une série de lacs de plus ou moins grande étendue qui communiquent avec le fleuve au cours de l'hivernage (Marigots ou lacs de Vely, d'Aoulik, de Dara, de Keur-Massene), certains d'entre eux ont des dimensions imposantes, plusieurs kilomètres de longueur pour le marigot de Vely.
Comme l'indique le tableau $n^{\circ} 2$, nous n'avons trouvé de mollusques d'eau douce que dans celui d'Aoulik, les autres ayant leurs eaux plus ou moins saumatres et leurs bords recouverts d'efflorescences salines déposées au cours de la baisse des eaux.

L'absence de chlorure de sodium dans le lac d'Aoulik s'expliquerait peut-être par la présence d'une importante résurgence en son centre, et par son éloignement du fleuve Sénégal avec lequel il communique par une chaîne de marigots où la marée remonte difficilement.

Il est à signaler d'autre part que dans cette région très sèche existent, de loin en loin, de petites mares de faible importance (quelques dizaines de mètres de diamètre) alimentées par des sources ne tarissant pas en saison sèche. Peu profondes et à fond vaseux, leur flore aquatique est représentée presque uniquement par des Nymphaea. Comme l'indique le tableau $n^{\circ} 2$, ce sont de petits gîtes à Bulinus guernei.

\section{Résultats obtenus à la dissection des mollusques d'eau douce récoltés dans les gîtes de la région du bas-fleuve Sénégal (Rosso).}

Ils sont consignés dans le tableau no 3 ef demandent les commentaires suivants :

Nous ne tiendrons compte que des formes lar-

TABLEAU no 3. - Résultats abtenus à la dissection des mollusques récoltés dans le Tagani et en région de Rosso

\begin{tabular}{|c|c|c|c|c|c|c|c|c|c|c|c|c|}
\hline \multirow{3}{*}{ Gîte } & \multirow{3}{*}{ Nature } & \multicolumn{11}{|c|}{ Espèces des mollusques disséqués - Formes larvaires - Pourcentage d'infestation. } \\
\hline & & \multicolumn{3}{|c|}{ B. truncatus } & \multicolumn{3}{|c|}{ B. senegolensis } & \multicolumn{3}{|c|}{ B. guernet } & \multicolumn{2}{|c|}{ Anisus sp. } \\
\hline & & Sch. & Par. & Strig. & Ech. & Xiph. & Div. & Seh. & Par. & Str. & Xiph. & Div. \\
\hline 2 & Guelta de Daber & 6 & & 15 & & & & & & & & \\
\hline 3 & Guelta de Sellembou & 16 & & 8 & & & & & & & & \\
\hline 4 & Marigot de Rosso & & & & 5 & 10 & 20 & 2 & 8 & 10 & 30 & 10 \\
\hline 5 & Loc d'Aoulik & & & & 20 & & & 2 & 10 & 15 & 15 & \\
\hline 6 & $\begin{array}{l}\text { Mare d̀ } 4 \mathrm{~km} \text { du lac } \\
\text { d'Aoulik }\end{array}$ & & & & & & & & 10 & 10 & & \\
\hline 7 & Marigot de Garak & & & & & & & & & & 10 & \\
\hline 8 & Marigot de Rosso (digue) & & & & & & & 5 & 10 & 30 & 20 & \\
\hline
\end{tabular}

Formes larvaires trouvées à la dissection des mollusques : Sch. = Schistosoma ; Par. = Poramphistomidoe ; Str. = Strigeidae $;$ Ech. $=$ Echinostomidae $;$ Xiph. = xiphidocercaires ; Div. $=$ divers. 
vaires de trématodes observées à la dissection des B. guernei, puisque chez B. senegalensis et Anisus sp. il n'a pas été possible de mettre en évidence celles de Schistosoma. Les seules parthenitae à sporocystes que nous ayons trouvées chez ces deux dernières espèces appartencient au groupe Strigeidoea avec furcocercaires pourvues d'un pharynx.

Aucun des B. guernei disséqués ne présentait de formes larvaires pouvant être rapportées à l'espèce Sch. haematobium ou à l'espèce Sch. bovis. Par contre, certains exemplaires (voir tableau $n^{0} 3$ ) étaient très fortement parasités par des furcocercaires et des éléments globuleux analogues à ceux de Schistosoma curassoni.

\section{DISCUSSION ET CONCLUSION}

Considérant les résultats obtenus au cours de ces enquêtes malacologiques, it nous semble permis de tirer les conclusions suivantes :

1. Les hôtés intermédiaires vecteurs de la bilharziose vésicale humaine et de la bilharziose des ruminants sont :

A. - En Haute-Casamance, Bulinus jousseaumei dont les principaux gîtes sont:

a) le bief amont de la Casamance de sa source jusqu'à Dianha-Malari, avec Kalda comme foyer très important de bilharziose vésicale ;

b) les marigots affluents de la rive gauche de la Casamance.

B. - Sur le plateau du Tagant, Bulinus truncatus rohifsi, avec comme foyer d'infestation des mares de montagnes appelées «gueltas», qui sont des gites permanents pour ce bulin.

\section{C. - En région du bas-fleuve Sénégal (Rosso),} Bulinus guernei, avec, comme gîtes permanents les marigots des environs de Rosso et quelques étangs et mares de la région nord-ovest de ce centre.

2. Au sujet du schistosome en cause, la dissection de plusieurs centaines de $B$. jousseaumel, $B$. truncatus rohlfsi et $B$. guernei, n'ont pas permis de mettre en évidence les furcocercaires et sporocystes de Sch. haematobium pas plus que les formes larvaires de Sch. bovis.
Par contre, parmi les bulins disséqués, de très nombreux exemplaires ont été trouvés porteurs de formes larvaires identiques à celles décrites récemment par GRÉTILLAT en 1962 pour Schistosoma curossoni (BRUMPT, 1931)*.

Or, comme nous venons de le démontrer au laboratoire, ce trématode est l'agent causal d'une bilharzıose commune à l'Homme et aux ruminants domestiques dans les régions de Dakar, du Cayor ef de la Haute-Casamance. II sembleraif donc que l'aire géographique de cette zoonose que nous avons appelée «Bilharziose Ouest-Africaine» ne serait pas uniquement limitée à la presqu'île du Cap Vert, mais s'étendrait à d'autres régions de l'Ouest-Africain.

Le principal « réservoir de parasites» étant le ruminant domestique (bøuf, mouton ef chèvre) qui héberge parfois plusieurs milliers de trématodes adultes, il est aisé de se rendre compte de l'importance de cette helminthiase en Haute Casamance où 30 à 40 p. 100 des bovins sont parasités et en Mauritanie (plateau du Tagant) où 70 à $80 \mathrm{p} .100$ des ruminants sont atteints massivement par cette parasitose.

Sur le plateau du Tagant, la rareté des points d'eau et leur faible importance ne font qu'augmenter les chances qu'a l'Homme de contracter cette helminthiase, En Haute Casamance, les baignades fréquentes des enfants durant toute l'année dans des eaux visitées tous les jours par les ruminants domestiques qui s'y abreuvent, expliqueraient le taux élevé de morbidité dô ò cette affection parasitaire.

\section{REMERCIEMENTS}

Nous remercions le Professeur G. RANSON du Museum National d'Histoire naturelle de

* Lors d'une récente étude sur l'épidémiologie de la bilharziose vésicale au Sénégal Oriental (GRÉTILLAT, 1961 , a) nous avons parlé de formes larvaires « pouvant appartenir à l'espèce Sch. hoematobium ». A cette époque, et malgré certaines particularités observées à la dissection des bulins, nous ne pouvions émettre de doule quant à l'identité de l'espèce en cause, les travaux déjà parus sur la bilharziose vésicale dans les pays de l'Ouest-Africain se référant toujours à Sch. haematobium. 
Paris d'avoir bien voulu déterminer pour nous, le matériel malacologique que nous avons récolté au cours de ces enquêtes épidémiologiques.

Une partie des recherches concernant les essais d'infestation expérimentale de bulins par des souches humaines de schistosomes en provenance de Kolda (Haute Casamance) a été finan- cée par un crédit accordé par l'Organisation Mondiale de la Santé à Genève.

\author{
Laboratorre Notional \\ de recherches vétérinaires \\ de Dakar-Hann (Rép. du Sénégal) \\ Service d'helminthologie
}

\section{SUMMARY}

\section{A contribution to the study of the epidemiology of humanandanimalbilharziosis in the upper casamance and in Mautiranie}

A series of snail surveys undertaken in Upper Casamance (Senegal) in the Tagant Plateau and in region of Rosso (Mauritania) in connection with human and animal bilharziosis gave the following results.

1. The intermediate hosts in the vesical form of human bitharziosis were

a) In Upper Casamance, Bulinus jousseoumei Dautzemberg, the habitat of which is noted in the river Casamance and its tributaries.

b) On the Tagant Plateau, B. truncatus rohlfsi Clessis, with habitats in the mountain pools.

c) In Ross region, B. guernei Dautzemberg, with habitats in tributaries in the River Senegal and also pools and ponds in the N.W.

2. From dissection of these molluscs (1.487 B. iousseaumei, 158 B. guernei and 200 B. truncotus roh/fsi) no furcocercaria or sporocysts of Sch. haefomobium or Sch. bovis were identified but on the other hand, many of the very special forms of Sch. curossoni Brumpt 1931, were identified. This is the common causal agent of West-African bilharziosis of both man and domestic animals.

This zoonosis appears to be widespread in all Senegal and a great part of Mauritania. Its presence has been proven by the identification of human schistosomiasis in parts of Dakar, Cayor and Upper Casamance. The principal reservoir of the parasites and disseminator of its eggs being the domestic ruminant.

\section{RESUMEN}

\section{Contribution al estudio de la epidemiologia de bilarciosis humanas y animales en el Senegal y Mauritania}

Una serie de sondeos epidemiológicos realizados por el servicio de Helmintología del Laboratorio nacional de Invesgaciones Veterinarias de Dakar, en Haute-Casamance (Republica del Senegal) sobre la mesetade Tagant y en la región de Rosso (República Islámica de Mauritania) sobre bilarciosis humana y animal, han permitido de llegar a los resultados siguientes :

10 Los huéspedes interemderios de la bilarciosis de la vejiga humana son :

a) En Alta Casamance, Bulinus jusseaumer Dautzemberg. que se le encuentra en el Casamance y sus zonas pantanosas.

b) En la meseta de Tagant, Bulinus truncatus rohlfsi (Clessin) que vive en las charcas de montaña.

c) En la region de Rosso, Bulinus guernei Dautzemberg, que se le encuentra en los terrenios pantanosos derlas horillas del Senegal, y de una manera accesoria sobre algunas charcas y lagos al NO. de Rosse.

$2^{\circ}$ A la disección de estos moluscos (1.487 B. jouseaumei, 158 B. guernei y 200 B. truncotus) no ha sido posible encontrar los furcocercaires y esporocistes de Sch. hematobiun o los de Sch. bovis, perosi, 


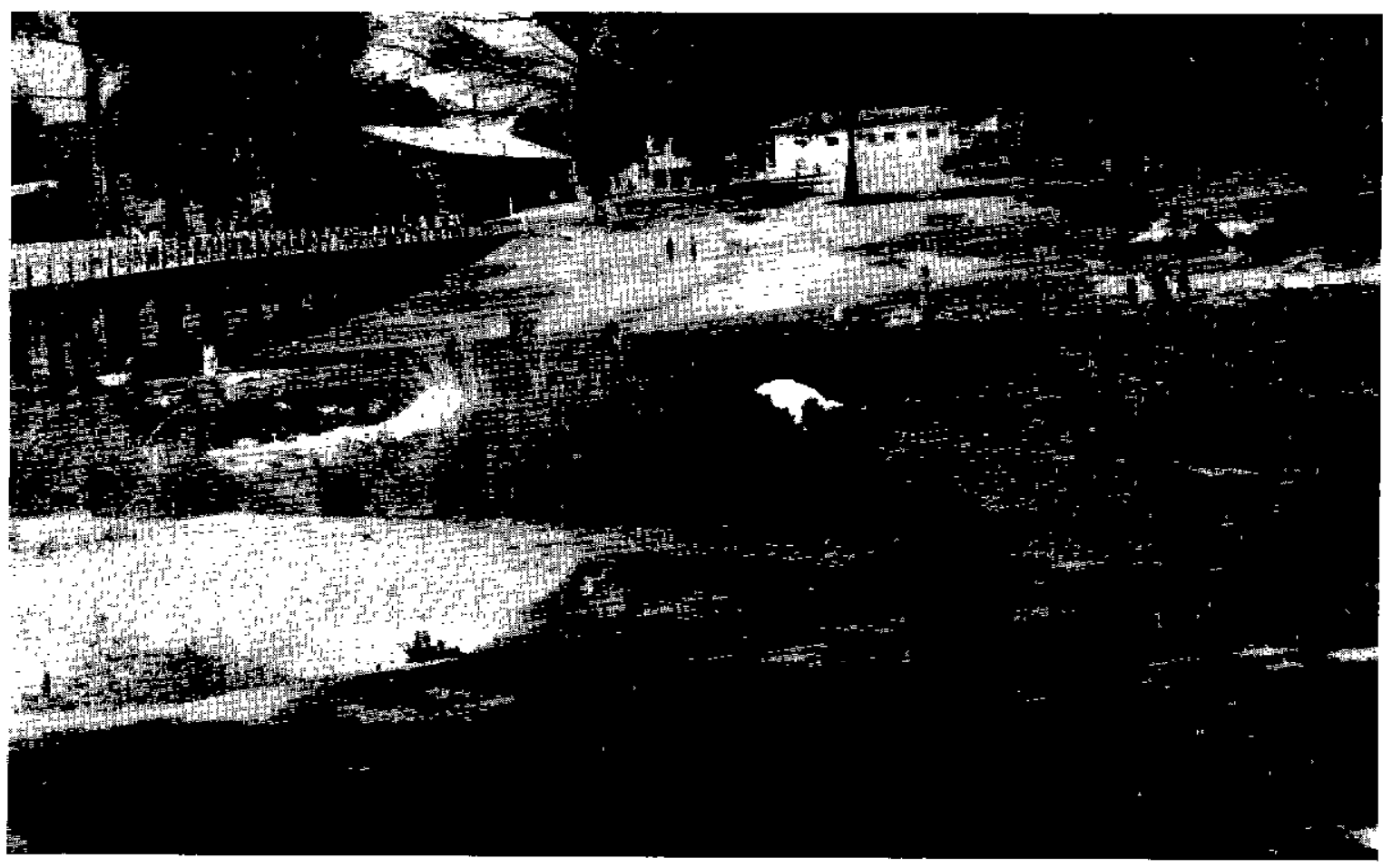

Photo no 1. - La Casamance au niveau du pont de Kolda (Haute Casamance).

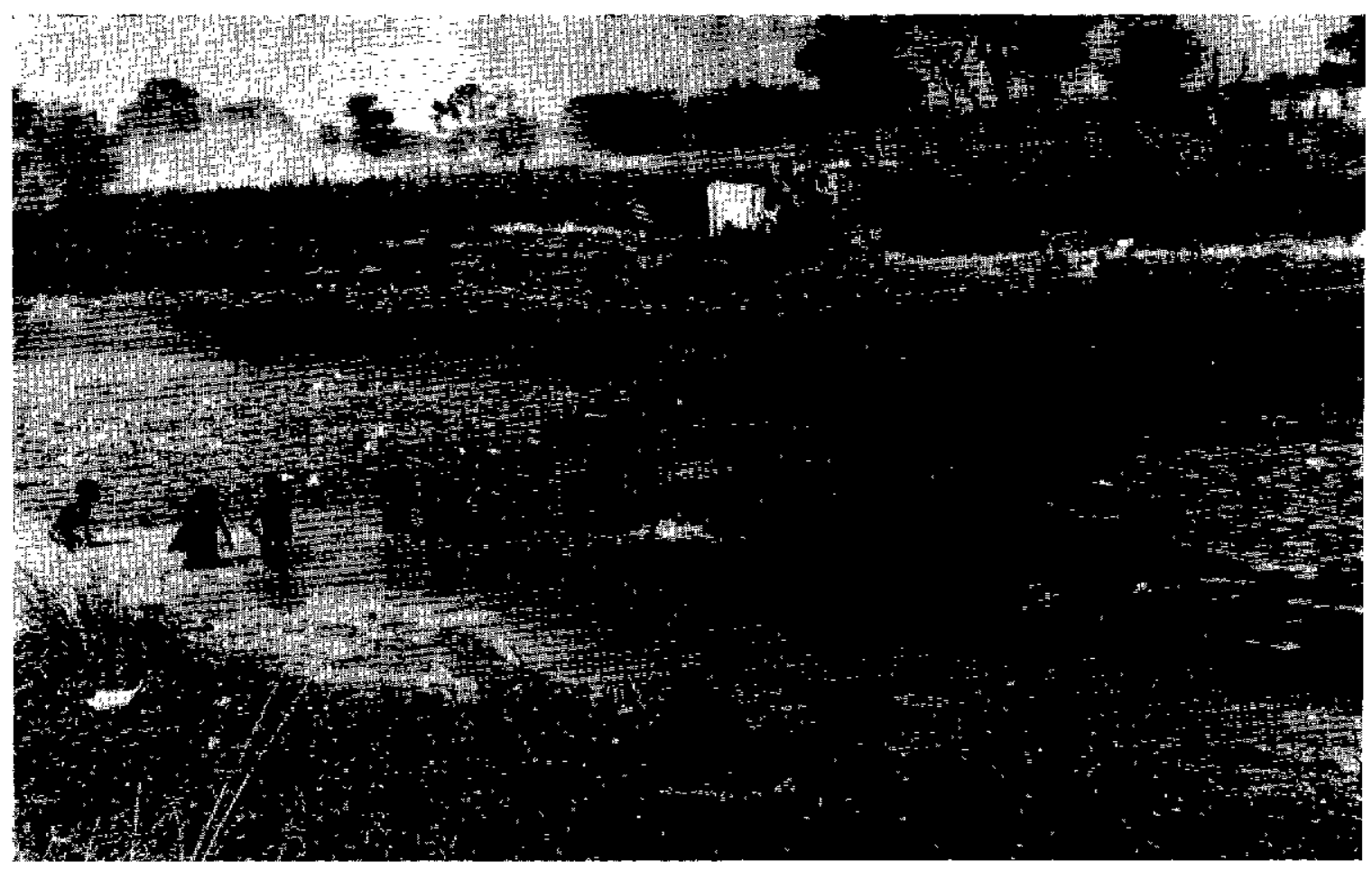

Photo no 2. - Gîtes à Bulinus jousseaumei en aval de Kolda. Lieu de baignade paur les enfants. 
sobre muchos, las formas larvarias muy particulares de Schistosoma curossoni agente causal de la Bilarciosis Oeste-Africana, helmintiasis común al hombre y a los ruminantes domésticos.

En conclusión, esta zoonosis, cuya existencia viene de demostrarse experimentalmente con la ayuda de cepas de schistosomas humanos de las regiones de Dakar, Cayor y Alta Casamance, y cuyo principal reservorio de parásitos y diseminador de huevos es el rumiante doméstico, tendría un área de repartición que se extendería a todo el Senegal y una gran parte de la Mauritania.

\section{BIBLIOGRAPHIE}

BOIRON (H.) et KOERBER (R.) (1947). - Contribution à l'étude de la bilharziose urinaire en A. O. F. Bull. Soc. Poth. exot., 40:118-25. BOUET ef ROUBAUD (1912). - Bilharziose au Dahomey et en Haute Casamance. Quelques observations biologiques sur le miracidium bilharzien. Bull. Soc. Path. exot., 5 : 837-42.

BRUMPT (E.) (1930). - Cycle évolutif complet du Schistosoma bovis. Infection naturelle en Corse ef infection expérimentale du Bulinus contortus. Ann. Parasit. hum. comp., $8: 17-50$.

BRUMPT (E.) (1931). - Description de deux Bilharzies de Mammifères africains : Schistosoma curossoni sp. inq. et Schistosoma rodhaini n. sp., Ann. Porasit. hum. comp., 9: 325-38.

BUTTNER (A.) (1958). - La bilharziose en Mauritanie. Influence de l'économie traditionnelle sur le cycle épidémiologique ef la résistance d̀ l'infestation. Sixième Congrès International de Médecine Tropicale et du Paludisme, Lisbonne, Institut de Médecine Tropicale (1958), 26 (Résumé).

DAGET (J.) (1961). — Le parc du Niokolo-Koba. Fasc. II, II-Mollusques d'eau douce, 13-29, Mém. Inst. Afr. Noire IFAN, Dakar.

DESCHIENS (R.) (1951, a). - Le problème sanitaire des bilharzioses dans les territoires de I'Union Française (Généralités et répartition géographique). Bull. Soc. Poth. exot., 44, 350-76.

DESCHIENS (R.) $(1951, b)$. - Le problème sanitaire des bilharzioses dans les territoires de I'Union Française (Thérapeutique, Prophylaxie). Bull. Soc. Poth. exot., 44: 667-88.
GAUD (J.) (1955). - Les Bilharzioses en Afrique occidentale et en Afrique centrale. Bull. org. mond. Santé, 44 : 209-58.

GRÉTILLAT (S.) (1961, a). --Epidémiologie de la bilharziose vésicale au Sénégal Oriental. Bull. arg. mond. Santé, 15: 459-66.

GRÉTILLAT (S.) (1961, b). — Note préliminaire sur l'épidémiologie de la distomatose bovine au Sénégal. Rev. Elev. Méd. vét. Pays trop., 14: 283-91.

GRÉTILLAT (S.) (1962, a). - Recherches sur le cycle évolutif du schistosome des Ruminants domestiques de l'Ouest-Africain (Schistosoma curassoni Brumpt, 1931). C. R. Acad. Sci., 255: 1.657-9.

GRÉTILLAT (S.) (1962, b). - Une nouvelle zoonose, la « Bilharziose Ouest-Africaine 》 à Schistosoma curassoni Brumpt, 1931, commune à l'Homme et aux Ruminants domestiques. C. R. Acod. Sci., $255: 1.805-7$.

GRÉTILLAT (S.) $(1962, c)$. - Ełude du cycle évolutif du Schistosome des Ruminants domestiques de l'Ovest Africain et confirmation de l'espèce Schistosoma curassoni Brumpi, 1931. Ann. Parasit, hum. comp. 37 : 556-68.

LEFROU (G.) (1933). - Présence de Bulinus dybowskyi au Sénégal. La diagnose des Bulinidae africains. Bull. Soc. Path. exot., $26: 1.099$.

MARILL (F. G.) (1960). - Rapport sur une enquête relative à l'épidémiologie des bilharzioses en Mauritanie. Secrétariat d'Etat aux relations avec les Etats de la Communauté. (Missions d'Experts) (avril-octobre 1960). 